\title{
Investigating liquid water distribution in Nafion polymer electrolyte membrane with terahertz imaging
}

\author{
Décio F. Alves de Lima, Rosa Letizia, Riccardo Degl'Innocenti, Richard Dawson, Hungyen Lin \\ Department of Engineering, Lancaster University, Lancaster LA1 4YW, United Kingdom
}

\begin{abstract}
Nafion ${ }^{\circledR}$ polymeric membranes are the most common electrolyte material for proton exchange membrane fuel cells (PEMFC), requiring adequate hydration in order to reach high proton conductivity. The relatively high attenuation of terahertz (THz) radiation of liquid water enables a contrast to be observed for inspecting water build-up in Nafion ${ }^{\circledR}$ membranes. This paper investigates the feasibility of a compact $\mathrm{THz}$ system using a $\mathbf{T H z}$ camera for liquid water imaging and quantification in a Nafion ${ }^{\circledR}$ polymeric membrane.
\end{abstract}

\section{INTRODUCTION}

$\mathrm{P}$ EMFC are electrochemical devices at the forefront of clean energy production for portable, transportation and, to a lesser extent, stationary applications. Water management is a key issue in these devices: during the fuel cell operation, protons emerge from the anodic hydrogen oxidation and flow across the polymer electrolyte membrane (PEM) to the cathode, combine with the electrons coming from the external load circuit and oxygen in the process to form water. Product water must be promptly removed from the cell, as it can flood catalytically active sites and hinder reactant mass transport. At the same time, the prime material for PEMs is Nafion ${ }^{\circledR}$, a perfluorinated ionomer membrane, which requires adequate hydration to achieve high proton conductivity and avoid material damage [1]. Therefore, a good balance of hydration needs to be maintained. As such, various techniques have been proposed to better understand the complex physical phenomena involving liquid water condensation and transport within the PEMFC. Examples of these techniques include MRI, neutron, $\mathrm{x}$-ray, infrared and direct visualisation [2]. In general, techniques such as MRI, neutron, and synchrotron x-ray imaging/tomography can provide in-situ information on liquid water transport through visually opaque components during cell operation at high spatial resolution, but are limited in terms of time resolution and facility availability. Direct visualisation and infrared imaging use compact and portable instruments with high time and spatial resolution, but in turn require optically transparent windows in a PEMFC in order to yield useful information on liquid water content. Particularly for inspecting water build-up and dynamics in Nafion membranes, techniques such as differential scanning calorimetry, gravimetric vapour sorption and electrochemical impedance spectroscopy have been used $[3,4]$. Whilst these measurements show good sensitivity to monitor hydration in Nafion membranes, they do not provide any spatially resolved information on liquid water distribution.

$\mathrm{THz}$ radiation can penetrate through optically opaque dielectric materials, such as polymers [5], while being strongly attenuated by liquid water [6] thus making $\mathrm{THz}$ imaging techniques attractive for non-destructive quality control applications [7-9]. With the recent availability of a compact, low-cost THz camera, as a first step toward PEMFC inspection, this paper explores the feasibility of a compact $\mathrm{THz}$ imaging system for inspecting liquid water in Nafion ${ }^{\circledR}$ PEMs. In particular, we assess system's ability to 1) quantify liquid water thickness; 2) estimate water weight; 3 ) spatially resolve water distribution across the membrane.

\section{METHODOLOGY}

A THz imaging system operating in transmission geometry consists an IMPATT $0.1 \mathrm{THz}$ source and a $16 \times 16$ array THz camera (TeraSense, USA), was realised. Nafion membranes of $180 \mu \mathrm{m}$ thickness (Alfa Aesar, UK) were imaged using the system yielding $\mathrm{THz}$ intensity images in the form of videos at $30 \mathrm{~Hz}$ framerate and at a $1.5 \mathrm{~mm}$ pixel size. To benchmark the $\mathrm{THz}$ images against gravimetric analysis, the mass of the hydrated membrane was monitored throughout the membrane dehydration process.

\section{RESULTS}

To demonstrate the imaging system's ability to quantify liquid water, $\mathrm{THz}$ transmission images were acquired through a custom-made liquid cell with pre-determined water thickness defined by the spacer thickness, where the relative intensities at regions of interest was found to be in a good agreement with the estimated intensities using Beer-Lambert law for a range of water thicknesses (Fig. 1).
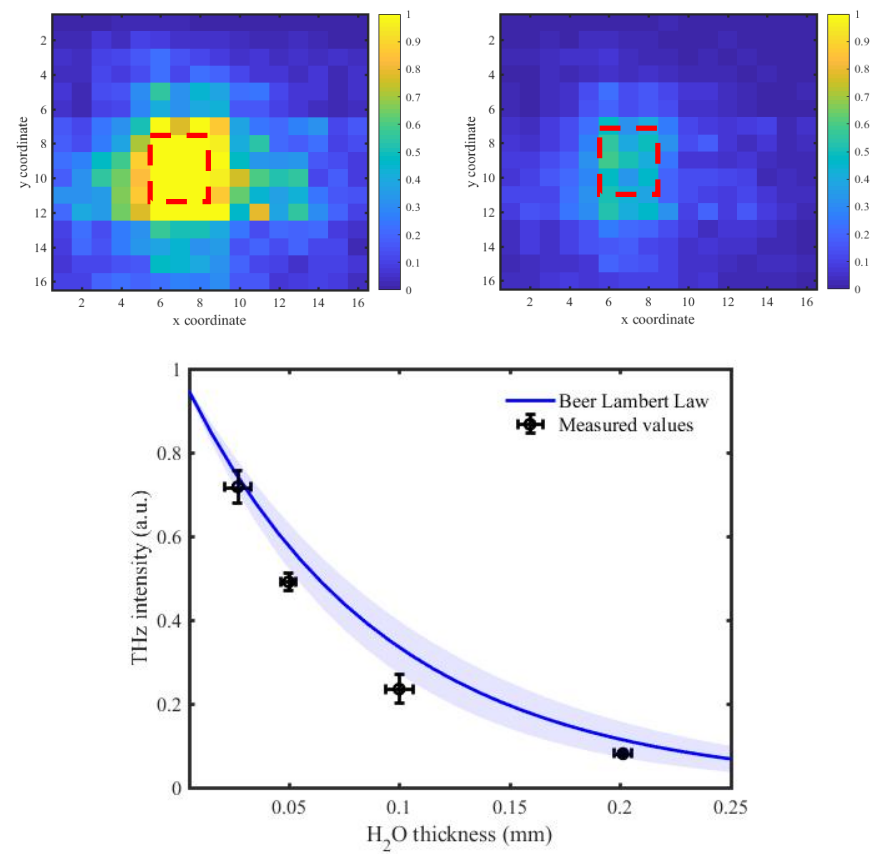

Fig. 1. THz liquid water content sensitivity analysis. Error bars account for spacer thickness variations (horizontal) and intensity standard deviation from 5 repeated measurements (vertical). Blue line and shade refer to mean and standard deviation between absorption coefficient values for the Beer Lambert Law.

Following liquid water quantification, the dehydration process of a Nafion PEM was monitored in ambient conditions, where liquid water content in the membrane was measured, converted to estimated weight, and benchmarked against 
gravimetric measurements (Figure 2). The water weight is estimated assuming constant water density $\left(1 \mathrm{~g} / \mathrm{cm}^{3}\right)$ and uniform water distribution across the entire membrane area within the THz measurement window. As can be seen, there is generally an agreement between the curves and the exponential decay nature of the process similar to the desorption work with Nafion membranes previously reported [10,11]. However, there are differences, such as the initial values and time constant. The former can be attributed to residual droplet presence on the Nafion surface from the hydration process, whose thickness can be outside of the system's sensitivity range $(25-200 \mu \mathrm{m})$, thus affecting the estimated weight values, while the latter is related to liquid water build-up on the testing rig that is outside of the sensor range.

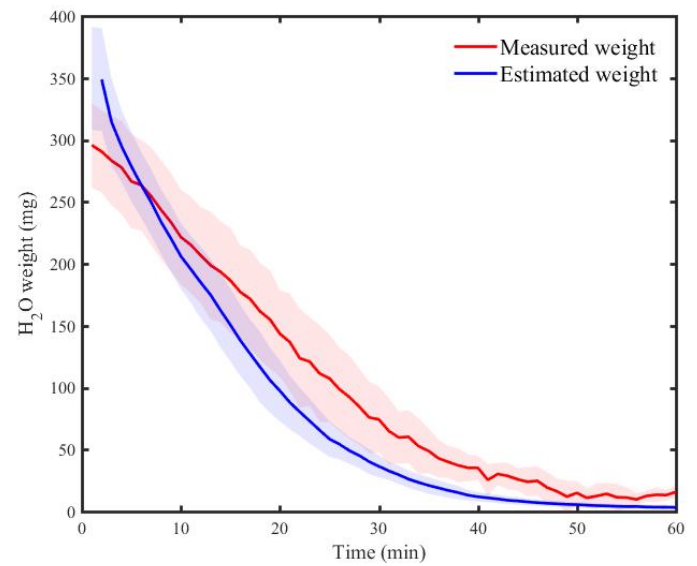

Fig. 2. Comparison of water weight loss estimated by THz measurement (blue) against gravimetric analysis (red). Lines and shades refer to mean and standard deviation, respectively, from 4 repeated experiments.

The $\mathrm{THz}$ imaging system also enables water to be spatially resolved across the membrane. In particular, Figure 3 shows the $\mathrm{THz}$ images of a partially-hydrated Nafion membrane, at three different time instants of a dehydration process: $1 \mathrm{~min} ; 30$ min and $120 \mathrm{~min}$. The figures all display two distinct areas: a hydrated area on the lower half, evidenced by the darker pixels caused by liquid water presence, and a dry area on the upper half, with much higher average THz transmission. Expectedly, with increasing time, the dark area shrinks due to water drying out. It is further possible to observe that water drying is not homogenous, with some areas drying at a faster rate than others. (a)

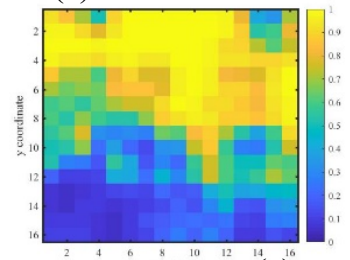

(c) (b)
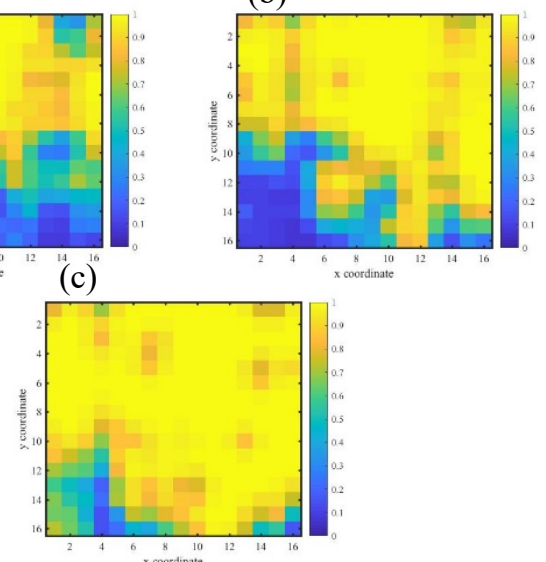

Fig. 3. Spatial differences of liquid water in the membrane at (a) $1 \mathrm{~min}$, (b) 30 min, (c) $120 \mathrm{~min}$ of a dehydration process.

\section{CONCLUSIONS}

In this study, we have investigated a compact $\mathrm{THz}$ transmission imaging system on quantifying and imaging liquid water content in Nafion PEM. In particular, we have assessed the system's sensitivity for quantifying liquid water, where we could reliably estimate water thickness between 25-200 $\mu \mathrm{m}$. Further, we have compared $\mathrm{THz}$ measurements against gravimetric measurements in a dehydration process where a general agreement can be observed despite assumptions uniform water distribution and constant water density. Finally, we have demonstrate the ability to qualitatively image spatial differences in liquid water across a Nafion membrane. As a whole, this technique has demonstrated to be an interesting alternative to liquid water imaging, which could prove to be useful in the quality control of Nafion PEM.

\section{ACKNOWLEDGEMENTS}

The authors acknowledge the EPSRC Impact Acceleration Account EP/R511560/1. HL also acknowledges the financial support from UK Engineering and Physical Sciences Research Council Research Grant EP/R019460/1. The authors would also like to thank Dr Massimo Peruffo and Dr Jonathan Sharman, from Johnson Matthey for helpful discussions.

\section{REFERENCES}

[1] M. Schalenbach, W. Lueke, W. Lehnert, D. Stolten, "The influence of water channel geometry and proton mobility on the conductivity of Nafion", Electrochim. Acta, vol. 214, pp. 362-369, 2016.

[2] A. Bazylak, "Liquid water visualisation in PEM fuel cells: A review", Int. J. Hydrog. Energy, vol. 34, pp. 3845-3857, 2009.

[3] B. Mecheri et al., "DSC and DVS investigation of water mobility in Nafion/Zeolite composite mebranes for fuel cell applications", J. Phys. Chem. C, vol. 116, no. 39, pp. 20820-20829, 2012.

[4] B. Legros et al.,"Electrochemical Impedance and Acoustic Emission Survey of Water Desorption in Nafion Membranes", Electrochem. Solid State Lett., vol. 12, no. 7, pp. B116B118, 2009.

[5] S. Wietzke et al.,"Terahertz spectroscopy on polymers: A review of morphological studies", J. Mol. Struct., vol. 1006, pp. 41-51, 2011.

[6] P. Thamboon et al., "Investigation of water distribution in proton exchange membrane fuel cells via Terahertz imaging”, Nucl. Instrum. Methods Phys. Res. $A$, vol. 637, pp. S161-S164, 2011.

[7] J. F. Federici, "Review of moisture and liquid detection and mapping using terahertz imaging", J. Infrared Millim. Terahertz Waves, vol. 33, no. 2, pp. 97$126,2012$.

[8] H. Lin et al. "Contactless graphene conductivity mapping on a wide range of substrates with terahertz time-domain reflection spectroscopy", Scientific Reports, vol. 7, pp. 10625, 2017.

[9] H. Lin et al. "Measuring of the intertablet coating uniformity of a pharmaceutical pan coating process with combined terahertz and optical coherence tomography in-line sensing", J. Pharm. Sci., vol. 106, no. 4, pp. 1075-1084, 2017

[10] P. W. Majsztriket al., "Water sorption, desorption and transport in Nafion membranes", J. Membrane Sci., vol. 301, pp. 93-106, 2007.

[11] M. Barclay Satterfield, J. B. Benziger, "Non-Fickian water vapour sorption dynamics by nafion membranes", J. Phys. Chem. B, vol. 112, no. 12, pp. 36933704,2008 . 